\title{
Aplicação do extrato de Abelmoschus esculentus (L.) Moench como floculante para a remoção de cor no tratamento de águas com corante têxtil
}

A indústria têxtil consome muita água em seus processos e gera efluentes que podem contaminar os corpos hídricos e afetar a vida aquática. A coagulação e a floculação são técnicas utilizadas no tratamento destes efluentes, e o quiabo pode ser utilizado como auxiliar de floculação. Assim, a partir de planejamento fatoria 23 buscou-se determinar as melhores concentrações, considerando a remoção de cor e geração de lodo como parâmetros. A partir de análise multivariada verificou-se que os melhores resultados de remoção de cor e formação de lodo foram alcançados com níveis inferiores, tanto de corante, quanto de coagulante. Além disso, foi possível concluir que a concentração de quiabo não foi significativa, indicando que é indiferente manter sua concentração no nível inferior ou superior, já que ambas as concentrações contribuem da mesma forma no processo de remoção de cor e formação de lodo.

\section{Application of abelmoschus esculentus (L.) Moench extract as floculant for color removal in water treatment with textile dye}

The textile industry consumes a lot of water in its processes and generates effluents that can contaminate water bodies and affect aquatic life. Coagulation and flocculation are techniques used to treat these effluents, and okra can be used as a flocculation aid. Thus, based on factorial planning 23 , it was sought to determine the best concentrations, considering the removal of color and generation of sludge as parameters. From multivariate analysis, it was found that the best results in removing color and forming sludge were achieved with lower levels of both dye and coagulant. Furthermore, it was possible to conclude that the concentration of okra was not significant, indicating that it is irrelevant to keep its concentration at the lower or higher level, since both concentrations contribute in the same way in the process of color removal and sludge formation.

Keywords: Flocculant; Okra; Multivariate Analysis.

Topic: Desenvolvimento, Sustentabilidade e Meio Ambiente

Reviewed anonymously in the process of blind peer
Received: 08/05/2020

Approved: 06/06/2020
Bruna Caroline de Lima

Universidade Tecnológica Federal do Paraná, Brasil http://lattes.cnpq.br/0792144643891275 lima.brunac@gmail.com

Gigliolla Caroline Biason Pinguelo

Universidade Tecnológica Federal do Paraná, Brasil http://lattes.cnpq.br/7172424959777519 gibiason@gmail.com

Laís Gimenes Vernasqui

Universidade Tecnológica Federal do Paraná, Brasil http://lattes.cnpq.br/9470290295051445 laisvernasqui@gmail.com
Nelson Consolin Filho (iD

Universidade Tecnológica Federal do Paraná, Brasil http://lattes.cnpq.br/5820606948533146

http://orcid.org/0000-0002-4847-0701 consolin@utfpr.edu.br

Marcilene Ferrari Barriquello Consolin (iD) Universidade Tecnológica Federal do Paraná, Brasi http://lattes.cnpq.br/3028205116451785

http://orcid.org/0000-0001-5499-2701 marcilenef@utfpr.edu.br

\section{Patrícia Valderrama}

Universidade Tecnológica Federal do Paraná, Brasil

http://lattes.cnpq.br/7543235815267877

http://orcid.org/0000-0002-6680-1260

pativalderrama@gmail.com
Flávia Vieira da Silva Medeiros

Universidade Tecnológica Federal do Paraná, Brasil http://lattes.cnpq.br/8298141172237555

http://orcid.org/0000-0001-6490-8876 flaviav@utfpr.edu.br

\section{Referencing this:}

LIMA, B. C.; PINGUELO, G. C. B.; VERNASQUI, L. G.; CONSOLIN FILHO, N.; CONSOLIN, M. F. B.; VALDERRAMA, P.; MEDEIROS, F. V. S. Aplicação do extrato de Abelmoschus esculentus (L.) Moench como floculante para a remoção de cor no tratamento de águas com corante têxtil. Revista Ibero-Americana de Ciências Ambientais, v.11, n.4, p.424-431, 2020. DOI: http://doi.org/10.6008/CBPC21796858.2020.004.0035 


\section{INTRODUÇÃO}

Os impactos mais significativos na indústria têxtil são o consumo de água e a geração de efluentes com elevada carga orgânica (amido, sabões, agentes engomantes) e de corantes (KUNZ et al., 2002). Sendo assim, a contaminação de corpos hídricos por esses efluentes com corantes têxteis, leva ao consumo do oxigênio dissolvido, afetando diretamente a vida aquática além de ser altamente prejudicial aos organismos fotossintetizantes (SHARKAWY et al., 2007).

A ampla variedade e complexidade dos efluentes têxteis, aliadas a determinações da legislação que exigem tratamentos eficientes, têm levado à criação de novas tecnologias que buscam o tratamento melhor e mais adequado, ponderando despesas, tempo e eficiência. A coagulação e a floculação são técnicas muito utilizadas neste caso. Essa operação é considerada como um pré-tratamento que tem como objetivo o condicionamento do despejo para o tratamento subsequente (VAZ, 2009).

Como alternativa aos polímeros sintéticos (sulfato de alumínio e cloreto férrico) normalmente empregados na etapa de coagulação/floculação, é possível utilizar polímeros naturais, que apresentam menor custo e não representam riscos à saúde humana. Quando usados em conjunto com o coagulante químico, esses polímeros podem reduzir a dosagem do coagulante metálico, promovendo a mesma eficiência de remoção de particulados e matéria orgânica natural, e possibilitando menores concentrações de alumínio residual no efluente tratado, o que implica em lodo gerado de menor volume e com maior facilidade de desaguamento (LIMA, 2007).

Alguns polímeros naturais já estudados e que apresentam desempenho satisfatório como auxiliares de floculação, são: malva (Malva sylvestris), quitosana (derivada da quitina), tanino (extraída da Acacia mearnsii), cactos (Cactaceae) (BELBAHLOUL et al., 2015; LEE et al., 2014). O quiabo, como auxiliar de floculação, tem boa performance e também maior possibilidade de utilização, devido à forma de comercialização deste vegetal, em que o consumidor rejeita o fruto maduro (LIMA, 2007).

Abelmoschus esculentus (L.) Moench (quiabo) (Figura 1) pertence à família Malvaceae, trata-se de uma planta arbustiva anual, com caule ereto esverdeado ou avermelhado, e atinge de 1 a 1,7 metros de altura. É uma hortaliça de clima quente e por isso é um dos cultivares mais bem adaptados ao clima tropical brasileiro. Temperaturas baixas causam danos aos frutos e impedem seu pleno crescimento (LIMA, 2007).

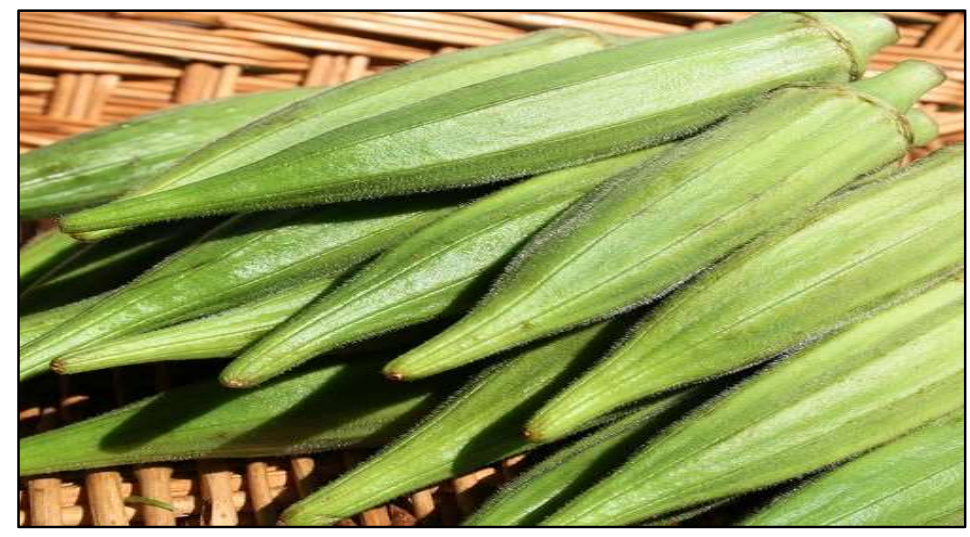

Figura 1: Frutos de Abelmoschus esculentus (quiabo), dos quais são extraídos polímeros que podem ser usados como auxiliar de coagulação e floculação. 
O quiabo, maduro é rejeitado pelo consumidor deste fruto, é estável, sendo produto apropriado para uso em tratamento de água e de efluentes como auxiliar de floculação por ser polímero aniônico. Pode ser usado na forma da mucilagem extraída da vagem ('baba' de quiabo) ou na forma de solução feita a partir do pó seco da vagem pulverizada, sendo esta última a forma mais simples de utilização (LIMA, 2007).

De acordo com Agarwal et al. $(2001 ; 2003)$, o quiabo possui um polissacarídeo aniônico, que pode ser usado como floculante e por ser polímero natural, é virtualmente biodegradável. Não é tóxico, é estável e tende a ser intensivamente usado no tratamento de águas de abastecimento, de esgoto doméstico e de efluentes de curtume. Com base no exposto, este trabalho teve como objetivo analisar a utilização de Abelmoschus esculentus (quiabo) como auxiliar de floculação na remoção de cor de soluções aquosa de corante alaranjado de metila, e também avaliar o lodo gerado neste processo.

\section{MATERIAIS E MÉTODOS}

Todos os ensaios foram realizados no Laboratório de Saneamento (LABSAN) da Universidade Tecnológica Federal do Paraná, Campus Campo Mourão. Para a realização deste trabalho, a metodologia foi dividida em quatro etapas: (1) Preparação das soluções; (2) Avaliações da remoção de cor e da geração de lodo; (3) Determinação das melhores condições pelo planejamento fatorial $2^{3}$; (4) Caracterização da solução de corante; (5) Caracterização do lodo gerado.

\section{Solução Aquosa de Alaranjado de Metila}

A solução aquosa de alaranjado de metila (fórmula química $=\mathrm{C}_{14} \mathrm{H}_{14} \mathrm{~N}_{3} \mathrm{NaO}_{3} \mathrm{~S} ; \mathrm{FW}=327,34 \mathrm{~g} / \mathrm{mol}$; natureza $=$ vermelho em pH na faixa $3,2-4,4 ; \lambda=458 \mathrm{~nm})$, nas concentrações de 0,05 e $0,15 \mathrm{~g} / \mathrm{L}(0,05$ e 0,15 g de corante dissolvido em 1 litro de água de torneira). O corante foi usado sem qualquer purificação adicional para preparar a solução. $O$ comprimento de onda foi determinado na concentração de $0,15 \mathrm{~g} / \mathrm{L}$ pelo método da varredura no espectrofotômetro. Posteriormente uma curva de calibração foi determinada para soluções com diferentes concentrações do corante utilizado.

\section{Soluções Utilizadas nos Ensaios de Coagulação/Floculação}

Sulfato de alumínio $\left(\mathrm{Al}_{2}\left(\mathrm{SO}_{4}\right)_{3}\right)$ foi utilizado como coagulante químico e hidróxido de cálcio $\left(\mathrm{Ca}(\mathrm{OH})_{2}\right)$ como alcalinizante. As soluções de coagulante químico e de alcalinizante foram preparadas com concentrações de $1 \%(\mathrm{v} / \mathrm{v})$. O coagulante químico foi aplicado diretamente na solução de corante a ser tratada, nas concentrações determinadas no planejamento experimental (0,5 e 1,5\%). Para a preparação da solução padrão do auxiliar de floculação (quiabo), foi dissolvido 0,1 g do quiabo seco e moído em $100 \mathrm{~mL}$ de água destilada em balão volumétrico, que resultou em uma solução $0,1 \%(\mathrm{~m} / \mathrm{v})$. As concentrações testadas foram determinadas no planejamento experimental (0,1 e 0,2\%).

\section{Ensaios de Coagulação e Floculação}

Para os ensaios de coagulação e floculação foi utilizado o equipamento de Jar Test modelo 218/LDB 
06 de seis provas da marca Nova Ética. Os ensaios foram realizados de acordo com o planejamento fatorial $2^{3}$, de acordo com a Tabela 1.0 tempo de mistura rápida foi de $1 \mathrm{~min}$ e o de mistura lenta, $10 \mathrm{~min}$, sendo as respectivas velocidades de rotação 180 e $30 \mathrm{rpm}$. A concentração de alcalinizante foi fixada em 5 ppm em todos os jarros.

Tabela 1: Planejamento fatorial $2^{3}$ para realização dos ensaios.

\begin{tabular}{llll}
\hline Ensaio & Concentração de Coagulante Químico & Concentração de Quiabo & Concentração de Corante \\
\hline 1 & $0,5 \%$ & $0,1 \%$ & $0,05 \mathrm{~g} / \mathrm{L}$ \\
2 & $1,5 \%$ & $0,1 \%$ & $0,05 \mathrm{~g} / \mathrm{L}$ \\
3 & $0,5 \%$ & $0,2 \%$ & $0,05 \mathrm{~g} / \mathrm{L}$ \\
4 & $1,5 \%$ & $0,2 \%$ & $0,05 \mathrm{~g} / \mathrm{L}$ \\
5 & $0,5 \%$ & $0,1 \%$ & $0,15 \mathrm{~g} / \mathrm{L}$ \\
6 & $1,5 \%$ & $0,1 \%$ & $0,15 \mathrm{~g} / \mathrm{L}$ \\
7 & $0,5 \%$ & $0,2 \%$ & $0,15 \mathrm{~g} / \mathrm{L}$ \\
8 & $1,5 \%$ & $0,2 \%$ & $0,15 \mathrm{~g} / \mathrm{L}$ \\
\hline
\end{tabular}

Após os processos de coagulação e floculação, o equipamento de Jar Test foi desligado, e as amostras mantidas em repouso por $10 \mathrm{~min}$, para que ocorresse a sedimentação do material floculado. A avaliação da geração de lodo foi realizada em relação ao volume gerado, utilizando-se de cones Imhoff, em todos os ensaios.

\section{Análise Multivariada dos Dados}

Os dados coletados neste trabalho foram avaliados por curvas de distribuição de probabilidade, construídos no software para identificar quais coeficientes de regressão foram significativos (BARROS NETO et al., 2003), MATLAB R2007b. Com os resultados obtidos destas curvas, foi possível a construção do modelo multivariado, para o qual também se utilizou o software MATLAB R2007b com as ferramentas do PLS (Partial Least Squares) Toolbox 5.2 (Eigenvector Research Inc.).

\section{Remoção de Cor}

A remoção de cor dos efluentes de indústrias têxteis representa um benefício bastante importante a ser alcançado, principalmente devido ao fato de a mesma estar relacionada à qualidade da água. Através do método espectrofotométrico para determinação de cor, descrito no Standard Methods for the Examination of Water and Wastewater (APHA, 2005), determinou-se a cor de amostras com base na elaboração de uma curva de calibração. Para a construção desta curva padrão, foi preciso definir primeiramente um comprimento de onda $(\lambda)$, adequado para as características das amostras a serem analisadas. A determinação deste comprimento de onda foi realizada através da leitura em espectrofotômetro, das absorbâncias para os diferentes comprimentos de onda. O comprimento de onda que apresentou o maior valor de absorbância, ou seja, o pico, foi utilizado para construir a curva de calibração da solução de alaranjado de metila.

A partir da definição do comprimento de onda, a curva foi construída para este comprimento de onda fixo, através da correlação entre valores de leituras de absorbância em espectrofotômetro e sua correlação com a concentração da substância que está sendo detectada, neste caso o corante. A cor foi medida por meio da leitura da absorbância em espectrofotômetro (modelo DR/5000 UV-VIS da HACH), no comprimento 
de onda de máxima absorbância da solução de corante, na faixa visível.

\section{RESULTADOS E DISCUSSÃO}

Para determinação da curva de calibração (Figura 3), foram feitas leituras de absorbância de soluções com oito concentrações diferentes $(0,3 ; 0,6 ; 0,9 ; 1,2 ; 1,5 ; 1,8 ; 2,1$ e 2,4 g/L - Figura 2) do corante. Com os resultados, foi possível construir o gráfico da absorbância pela concentração e consequentemente, obter a equação da reta de ajuste linear e do coeficiente de correlação $\left(R^{2}\right)$.

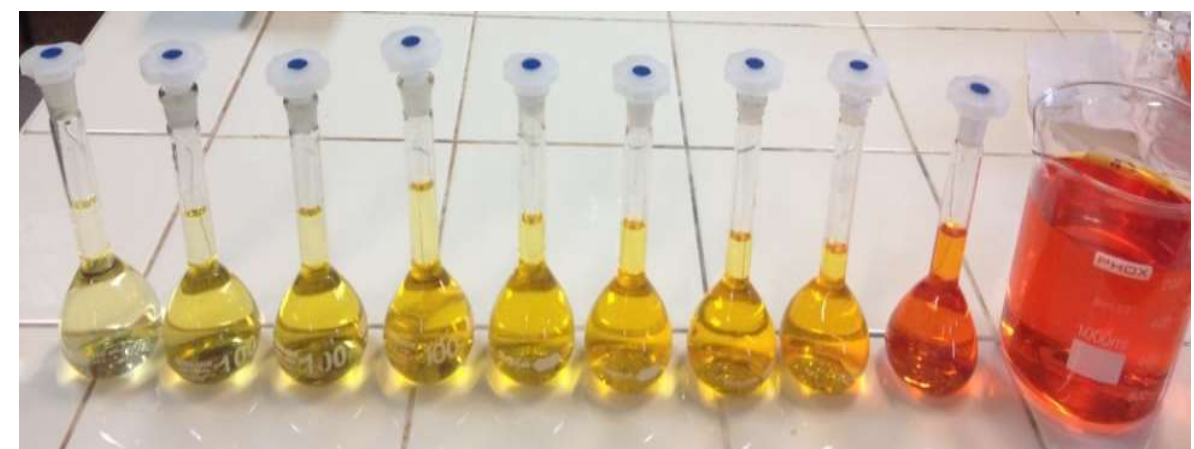

Figura 2: Balões volumétricos com as soluções de diferentes concentrações de corante, utilizados para a determinação da curva da calibração.

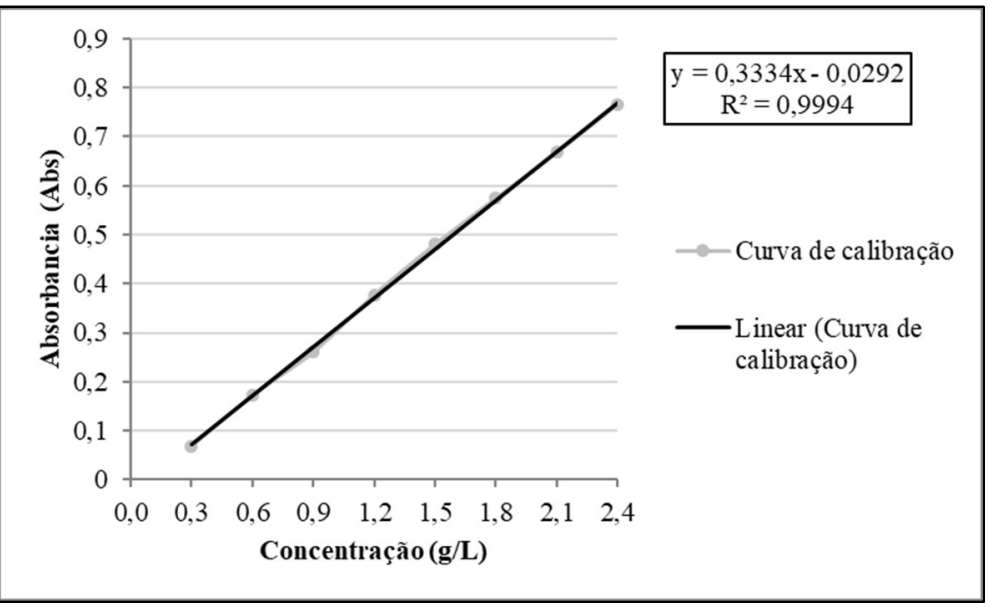

Figura 3: Curva de calibração e a reta de ajuste linear, determinadas pela varredura realizada com o alaranjado de metila no espectrofotômetro.

Com os resultados da absorbância e determinação da curva de calibração, foi possível comprovar o que já se esperava, a absorbância aumenta à medida que a concentração da solução aumenta e a definição da reta de ajuste linear e o coeficiente de correlação muito próximo de 1 (um), comprovam essa afirmação. Nas Figuras 4 e 5 são apresentadas as curvas das distribuições de probabilidade normalizadas para os efeitos padronizados para se avaliar cada fator e suas interações no processo de tratamento da solução aquosa de alaranjado de metila utilizando o quiabo como auxiliar de floculação.

No caso da cor (Figura 3), o valor positivo do coeficiente 2 (Coagulante Químico) significa que a cor se torna mais intensa com a concentração maior de coagulante químico. 0 valor negativo do coeficiente 4 (Corante) significa que a cor se torna menos intensa com a concentração menor do corante. No lodo (Figura 5 ), o valor negativo do coeficiente 2 (CQ) significa que a cor se torna menos intensa com a concentração menor de coagulante químico. O efeito do floculante (Quiabo - Q) sozinho não foi importante no processo, 
porém a interação desse floculante com ele mesmo se mostrou significativa (coeficiente 10).

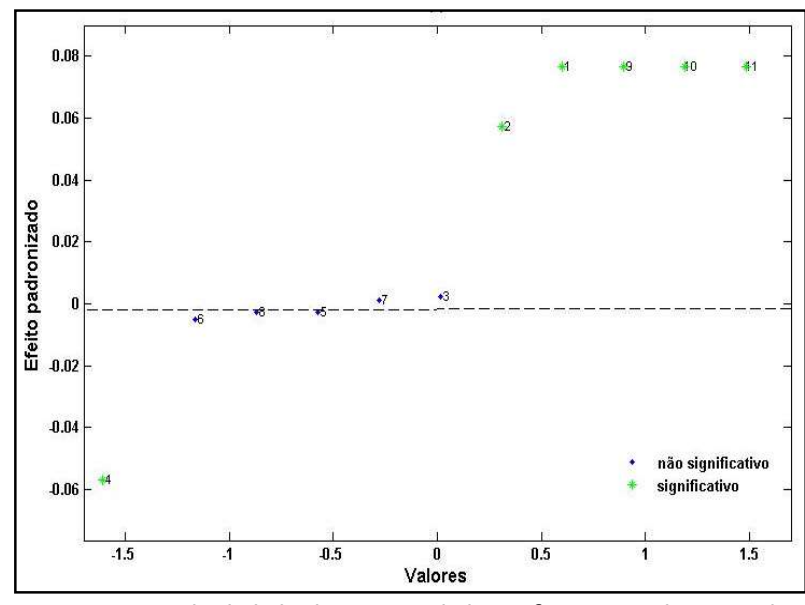

Figura 4: Probabilidade normal dos efeitos padronizados na remoção de cor (1-média, 2-Coagulante Químico-CQ, 3-Floculante-Q, 4-Corante-C, 5-CQ.Q, 6-CQ.C, 7-Q.C, 8CQ.Q.C, $\left.9 C Q^{2}, 10-Q^{2}, 11-C^{2}\right)$.

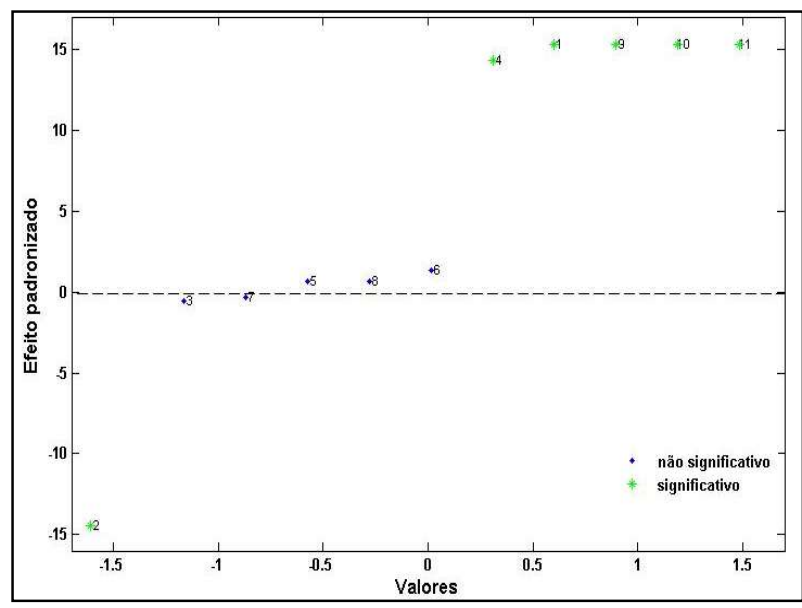

Figura 5: Probabilidade normal dos efeitos padronizados na formação de lodo. (1-média, 2-Coagulante QuímicoCQ, 3-Floculante-Q, 4-Corante-C, 5-CQ.Q, 6-CQ.C, 7-Q.C, 8-CQ.Q.C, $\left.9 C Q^{2}, 10-Q^{2}, 11-C^{2}\right)$.

Após realizar uma triagem dos fatores e verificar quais foram significativos no processo, foi possível escrever uma equação simplificada para o modelo matemático na remoção de cor (Equação 1) e na formação de lodo (Equação 2) e realizar uma análise da superfície de resposta para se obter a máxima remoção de cor e formação de lodo.

$$
\begin{aligned}
& y=0,0765+0,00573 \cdot C Q-0,0568 \cdot C+0,0765 \cdot C Q^{2}+0,0765 \cdot Q^{2}+0,0765 \cdot C^{2} \\
& y=15,3281-14,4383 \cdot C Q+14,3117 \cdot C+15,3281 \cdot C Q^{2}+15,3281 \cdot Q^{2}+15,3281 \cdot C^{2}
\end{aligned}
$$

Em ambos os casos, como as concentrações de coagulante químico (CQ) e corante (C) foram significativas, fixou-se a concentração do floculante $(Q)$ nos níveis inferior e superior para a construção da superfície de resposta. As superfícies de resposta para a cor remanescente no processo são apresentadas nas Figuras 6 e 7.

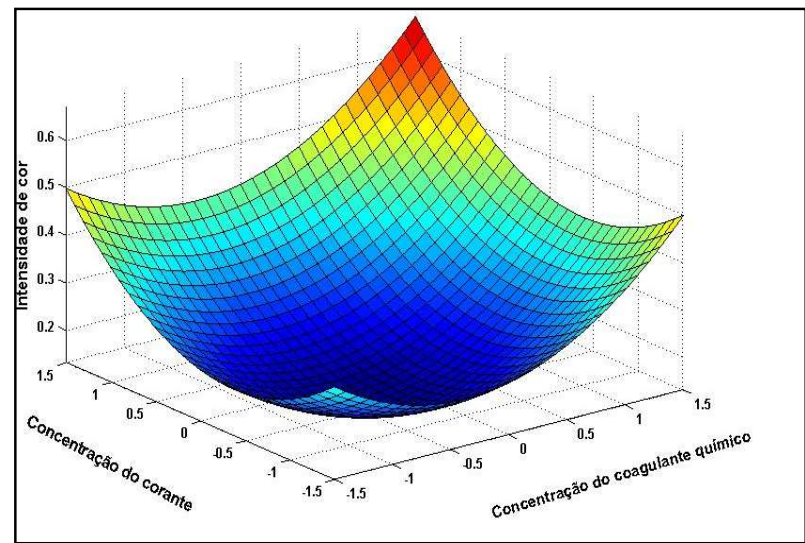

Figura 6: Superfície de resposta para o modelo obtido na remoção de cor quando a concentração de quiabo estava no nível superior.

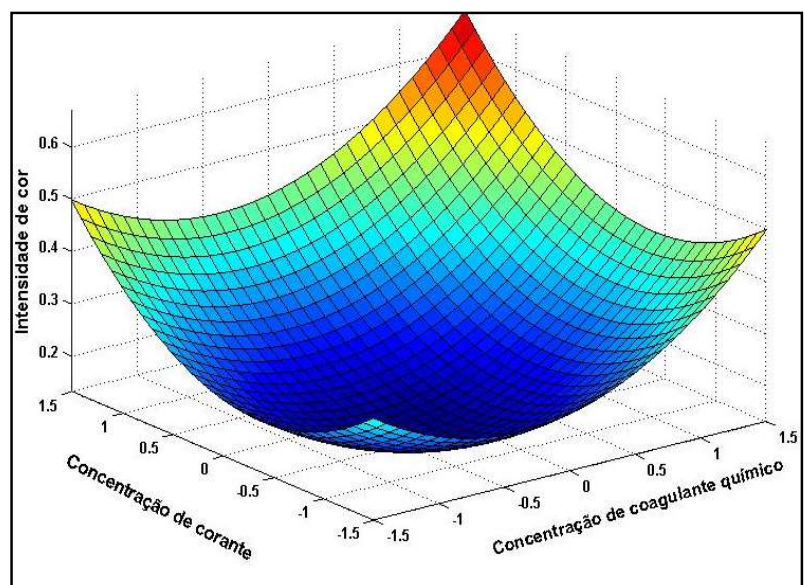

Figura 7: Superfície de resposta para o modelo obtido na remoção de cor quando a concentração de quiabo foi mantida no nível inferior.

Por meio das superfícies de respostas, fica comprovado que a concentração do auxiliar de floculação não influencia o processo de remoção de cor, uma vez que as superfícies são praticamente idênticas. Sendo 
assim, é vantajosa a utilização de concentrações menores do auxiliar de floculação, uma vez que a eficiência de remoção não se altera. As superfícies de resposta para a quantidade de lodo gerada no processo são apresentadas nas Figuras 8 e 9.

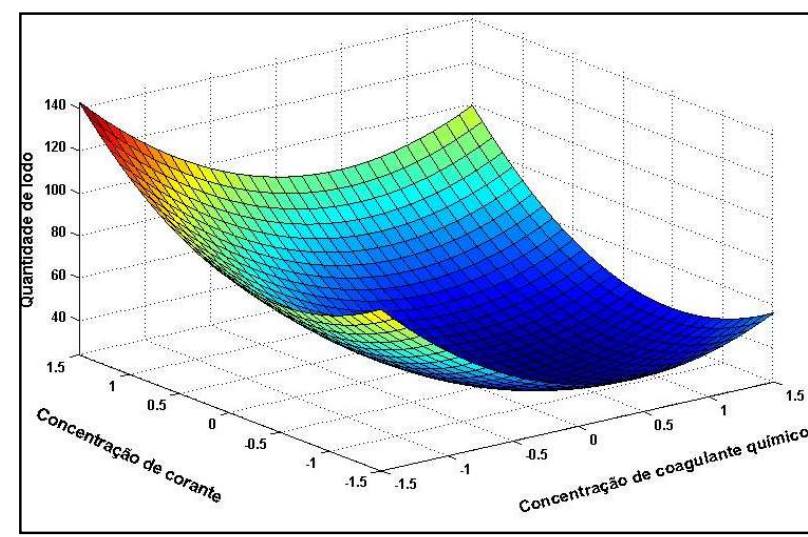

Figura 8: Superfície de resposta para o modelo obtido na formação de lodo quando a concentração de quiabo estava no nível superior.

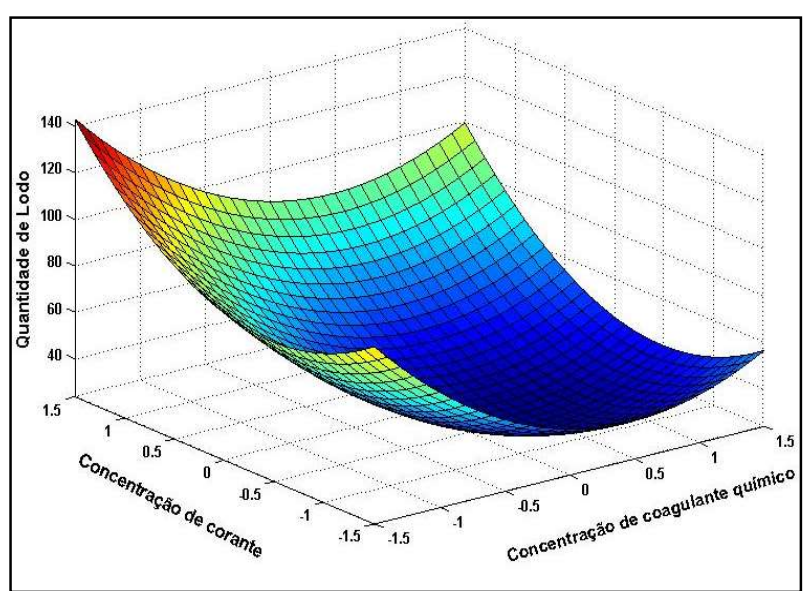

Figura 9: Superfície de resposta para o modelo obtido na formação de lodo quando a concentração de quiabo foi mantida no nível inferior.

A maior quantidade de lodo é formada quando as concentrações de corante e coagulante químico encontram-se nos níveis inferiores. Nesse caso também não existe influência significativa da concentração do auxiliar de floculação no processo, e as superfícies são praticamente idênticas.

\section{CONCLUSÕES}

Com os resultados obtidos com o planejamento fatorial, foi possível concluir que melhores valores de remoção de cor e formação de lodo são adquiridos com as concentrações de corante e coagulante químico nos níveis inferiores de concentração. A concentração de quiabo no processo não foi significativa, o que não significa que a sua presença não seja importante. Nesse caso foi indiferente manter a concentração de quiabo no nível inferior ou superior, ambas concentrações contribuem da mesma forma no processo de remoção de cor e formação de lodo.

Além disso, a ferramenta da otimização multivariada se mostrou bastante eficaz, facilitando a determinação da melhor condição experimentada, em que ocorresse a máxima remoção de cor e máxima produção de lodo. O auxiliar de floculação na concentração mais alta se mostrou mais eficiente no tratamento. $\mathrm{O}$ que demonstra que os estudos referentes a aplicação de quiabo como auxiliar de floculação na remoção de cor de efluentes têxteis, devem ser continuados.

\section{REFERÊNCIAS}

AGARWAL, M.; SRINIVASAN, R.; MISHRA, A.. Study on Flocculation Efficiency of Okra Gum in Sewage Waste Water Macromolecular materials and Engineering, v.286, n.9, 2001.

AGARWAL, M.; RAJANI, S.; MISHRA, A. R.. Utilization of Okra Gum for Treatment of Tannery Effluent. International
Journal of Polymeric Materials, v.53, n.11-12, 2003. DOI: https://doi.org/10.1080/714975900

APHA. American Public Health Association. Standard Methods for the Examination of Water and Wastewater. 20th. Washington: APHA, 2005. 
BARROS NETO, B.; SCARMINIO, I. S.; BRUNS, R. E.. Como

fazer experimentos: Pesquisa e desenvolvimento na ciência e na indústria. 2 ed. Campinas: Unicamp, 2003.

BELBAHLOUL, M.; ZOUHRI, A.. Bioflocculants extraction from Cactaceae and their application in treatment of water and wastewater. Journal of Water Process Engineering, v.7, p.306-313, 2015

KUNZ, A.; PERALTA-ZAMORA, P.; MORAES, S. G.; DÚRAN, N.. Novas Tendências no Tratamento de Efluentes Têxteis. Química nova, v.25, n.1, p.78-82, 2002.

LEE, C. S.; ROBINSON, J.; CHONG, M. F.. A review on application of flocculants in waste water treatment. Process
Safety and Environmental Protection, v.92, p.489-508, 2014.

LIMA, G. J. A.. Uso de Polímero Natural do Quiabo como Auxiliar de Floculação e Filtração em Tratamento de Água e Esgoto. Dissertação (Mestrado em Engenharia Ambiental) Universidade do Estado do Rio de Janeiro, Rio de Janeiro, 2007.

SHARKAWY, E. A. E.; SOLIMAN, A. Y.; AMER, K. M. A.. Comparative study for the removal of methylene blue via adsorption and photocalytic degradation. Journal of Colloid and Interface Science, v.310, p.498-508, 2007.

A CBPC - Companhia Brasileira de Produção Científica (CNPJ: 11.221.422/0001-03) detém os direitos materiais desta publicação. Os direitos referem-se à publicação do trabalho em qualquer parte do mundo, incluindo os direitos às renovações, expansões e disseminações da contribuição, bem como outros direitos subsidiários. Todos os trabalhos publicados eletronicamente poderão posteriormente ser publicados em coletâneas impressas sob coordenação da Sustenere Publishing, da Companhia Brasileira de Produção Científica e seus parceiros autorizados. Os (as) autores (as) preservam os direitos autorais, mas não têm permissão para a publicação da contribuição em outro meio, impresso ou digital, em português ou em tradução. 